\title{
Expert Opinion on Pegloticase with Concomitant Immunomodulatory Therapy in the Treatment of Uncontrolled Gout to Improve Efficacy, Safety, and Durability of Response
}

\author{
John K. Botson ${ }^{1} \oplus \cdot$ Herbert S. B. Baraf ${ }^{2} \cdot$ Robert T. Keenan $^{3} \cdot$ John Albert $^{4} \cdot$ Karim R. Masri $^{5} \cdot$ Jeff Peterson $^{6}$. \\ Christianne Yung $^{7} \cdot$ Brigid Freyne $^{8} \cdot$ Mona Amin $^{9} \cdot$ Abdul Abdellatif $^{10} \cdot$ Nehad Soloman $^{11} \cdot$ N. Lawrence Edwards ${ }^{12}$. \\ Vibeke Strand ${ }^{13}$
}

Accepted: 6 December 2021 / Published online: 15 February 2022

(c) The Author(s) 2022

\begin{abstract}
Purpose of Review Gout is a systemic disease from which some patients develop numerous painful tophi that adversely affect quality of life and functionality. Some patients treated with oral urate-lowering therapy are unable to maintain serum urate levels below $6 \mathrm{mg} / \mathrm{dL}$, and these patients, thus classified as having refractory or uncontrolled gout, often require therapy with pegloticase to reduce symptoms and tophaceous burden. The objective of this expert opinion review is to summarize the available evidence supporting the use of concomitant immunomodulators with pegloticase to prevent development of anti-drug antibodies (ADAs) when treating patients with uncontrolled gout.

Recent Findings Emerging evidence suggests that adding an immunomodulator to pegloticase therapy can substantially increase response rates to double those observed in phase 3 randomized controlled trials.

Summary The combination of immunomodulation with pegloticase should be considered in routine clinical practice to improve durability of response, efficacy, and safety among patients with uncontrolled gout who otherwise have limited therapeutic options.
\end{abstract}

Keywords Antidrug antibodies $\cdot$ Gout $\cdot$ Immunogenicity $\cdot$ Immunomodulation $\cdot$ Pegloticase

\section{Introduction}

The use of biologic agents has led to important treatment advances for multiple rheumatologic conditions. Historically, biologic agents have been associated with the formation of anti-drug antibodies (ADAs), which may result in loss or lack of efficacy and/or adverse effects $[1,2 \bullet]$. The coadministration of biologic agents with immunomodulating therapies that inhibit proliferation of activated lymphocytes has been shown to prevent or minimize ADA development, improve response, and lengthen therapy duration [3-6]. This approach has increased in use among clinical rheumatologists $[1,2 \bullet, 7,8]$ and has shown success when treating patients with chronic refractory gout, hereafter referred to

This article is part of the Topical Collection on Crystal Arthritis

John K. Botson

jbotson@opaak.com

Extended author information available on the last page of the article as uncontrolled gout, with the biologic agent, pegloticase [8-13, 14・•, 15].

Uncontrolled gout poses a burden for patients and a challenge for treating physicians. Uncontrolled gout affects a small subset of the 9.2 million individuals with gout in the USA [16, 17]. Gout is a progressive, systemic, crystal deposition disease arising from persistent elevations of serum urate (SU) [18]. Imaging studies have shown urate crystal deposits in joints, soft tissues, cartilage, and internal organs [18-22]. Dual-energy computed tomography has demonstrated extra-articular monosodium urate (MSU) deposition, or tophi, where clinical examination may not [23-25]. Recent guidelines recommend that patients with gout maintain SU levels $\leq 6 \mathrm{mg} / \mathrm{dL}$ to eliminate crystalline deposits and resolve clinical manifestations of gout [26]. Patients with uncontrolled gout are either unable to achieve or maintain SU levels $\leq 6 \mathrm{mg} / \mathrm{dL}$ or derive adequate clinical benefit due to their extensive urate burden despite treatment with oral urate-lowering therapies [26, 27]. Extensive literature has shown that the degree of SU lowering is proportional to 
the speed by which urate deposits can be eliminated [28-30]. Treatment with pegloticase, the only biologic therapy currently approved in the USA for treatment of uncontrolled gout, has been demonstrated to resolve tophi and improve pain and function [28, 31-34]. The 2020 American College of Rheumatology (ACR) guidelines recommend pegloticase as third-line treatment for patients with uncontrolled gout, including those with chronic tophaceous gouty arthropathy for whom SU thresholds well below $6 \mathrm{mg} / \mathrm{dL}$ may be preferable [26].

This paper reviews the evidence supporting the use of concomitant immunomodulators with pegloticase to prevent development of ADAs when treating patients with uncontrolled gout with pegloticase. Our assessment was guided by identification of immunogenicity as the primary factor leading to loss of response in phase 3 randomized controlled trials (RCTs) of pegloticase and recent publications demonstrating improvement due to prevention of ADA formation with use of antiproliferative agents [31,33, 35].

\section{Efficacy and Safety of Pegloticase}

Pegloticase, a pegylated recombinant mammalian uricase, is a US Food and Drug Administration-approved medication for the treatment of uncontrolled gout $[34,36]$. This enzyme catalyzes the conversion of uric acid to allantoin, a compound that is readily removed from the body through renal excretion [36]. Previously published RCTs of pegloticase in patients with uncontrolled gout intolerant to conventional urate-lowering therapies with $\mathrm{SU}$ levels $\geq 8.0 \mathrm{mg}$ / $\mathrm{dL}$ reported significant tophi resolution, reduced gout flares, and improvements in tender joint counts and patient reported health-related quality of life $[37,38]$. The primary end point of these RCTs was the proportion of patients who achieved SU levels $<6.0 \mathrm{mg} / \mathrm{dL}$ for $\geq 80 \%$ of the time during months 3 and 6 of active treatment versus placebo [37]. Overall, $42 \%$ who received biweekly pegloticase were responders [37].

A secondary end point was complete resolution of prespecified tophi at 6 months, which was confirmed in $45 \%$ of subjects who received pegloticase compared with $7 \%$ with placebo $(p=0.002)$ [37]. Across pooled study groups, infusion reactions ( $26 \%$ vs $5 \%$ of patients, respectively) were the second most common adverse event; gout flares being most common [30, 36]. Investigator-identified serious infusion reactions, none of which resulted in hospitalizations or death, occurred in $5 \%$ of subjects who received biweekly pegloticase vs none with placebo [31].

The relationship between therapeutic responses to pegloticase, development of ADAs, and risk of infusion reactions was determined in a post hoc analysis of the phase 3 RCTs [31]. In the phase 3 RCTs, ADAs were detected in $89 \%$ of patients at least once during the 6-month studies. High-titer
ADAs ( $>1: 2430)$ were detected in $41 \%$ of subjects and were significantly associated with loss of therapeutic response $(p<0.001)$ [31] and subsequent risk of infusion reactions with continued infusions [31, 33, 39]. Results from these analyses indicated that monitoring for elevated pre-infusion SU levels could identify patients at risk for development of ADAs and thus infusion reactions. These recommendations are included in the prescribing information $[35,36]$.

\section{Anti-drug Antibodies and Immunomodulation}

Pegloticase is an effective therapy in uncontrolled gout, yet its use is limited in a substantial proportion of patients by the development of high-titer-binding ADAs directed against the polyethylene glycol (PEG) moiety of the overall molecular structure [35, 37]. Formation of pegloticase ADAs has no direct inhibitory effect on uricase activity; instead, ADAs increase clearance of the molecule to a degree that its beneficial effects on SU levels is reduced and risk of infusion reactions increased $[33,35,39]$. Although the risk of infusion reactions due to ADAs can be substantially lowered by using the SU monitoring protocol and withdrawing treatment when SU levels increase to $>6 \mathrm{mg} / \mathrm{dL}[31,33,35,39]$, a more effective approach is to abrogate formation of ADAs [1]. Abrogation by cotreatment with antiproliferative agents has been the subject of a growing body of literature. Such agents, traditionally referred to as conventional disease-modifying antirheumatic drugs (cDMARDs), include methotrexate (MTX), azathioprine (AZA), leflunomide (LEF), and mycophenolate mofetil (MMF). These cDMARDs, which reversibly inhibit purine or pyrimidine synthesis are better tolerated and more efficacious than traditional immunosuppressants [1, 3-6, 8-12, 14••].

Various case reports, proof-of-concept case studies, and ongoing RCTs have shown favorable outcomes by combining pegloticase with immunomodulation in patients with uncontrolled gout. Response rates from these studies range from 60 to $100 \%$ (Table 1), nearly double the reported $42 \%$ response with pegloticase monotherapy in phase 3 RCTs $[8-13,14 \bullet \bullet, 15,40 \bullet \bullet]$.

Case reports by Berhanu et al. [9] and Freyne [11] first demonstrated successful treatment with pegloticase and concomitant use of AZA and MMF/cyclosporine, respectively, in patients with uncontrolled gout. Of note, the patient described in Berhanu et al. [9] experienced 2 increases in SU levels $(1.0 \mathrm{mg} / \mathrm{dL}$ and $6.2 \mathrm{mg} / \mathrm{dL}$, respectively) attributable to AZA nonadherence, which rapidly reversed to $<1.0 \mathrm{mg} /$ $\mathrm{dL}$ after reinstitution of AZA [9].

A proof-of-concept case series conducted by Botson and Peterson evaluated the utility of co-administration of oral MTX (15 mg weekly) with pegloticase ( $8 \mathrm{mg}$ 
Table 1 Summary of data: pegloticase and immunomodulation

\begin{tabular}{|c|c|c|c|}
\hline Author & Type of study ${ }^{a}$ & IMT and run-in (time frame) ${ }^{\mathrm{b}}$ & Responders, $n / N(\%)$ \\
\hline Berhanu et al. [9] & Case report & Azathioprine (2 weeks) & $1 / 1(100)$ \\
\hline Freyne [11] & Case report & $\begin{array}{l}\text { Mycophenolate mofetil and cyclosporine } \\
\text { (background therapy) }\end{array}$ & $1 / 1(100)$ \\
\hline Botson et al. $[14 \bullet \bullet]$ & Proof-of-concept case series & Methotrexate (4 weeks) & $10 / 10(100)$ \\
\hline Albert et al. [8] & Case series & Methotrexate (14-35 days) ${ }^{\mathrm{c}}$ & $8 / 10(80)$ \\
\hline Bessen et al. [10] & Case series & $\begin{array}{l}\text { Methotrexate, azathioprine, or cyclo- } \\
\text { sporine (at first infusion) }\end{array}$ & $7 / 7(100)$ \\
\hline Masri et al. [12] & Retrospective case study & Leflunomide (variable) & $4 / 6(67)$ \\
\hline Botson et al. [15] & Open-label pilot study (MIRROR) & Methotrexate (4 weeks) & $11 / 14(79)$ \\
\hline Rainey et al. [13] & Open-label pilot study (TRIPLE) & Azathioprine (2 weeks) & $6 / 10(60)^{\mathrm{d}}$ \\
\hline Khanna et al. $[40 \bullet \bullet]$ & Randomized controlled trial (RECIPE) & Mycophenolate mofetil (2 weeks) & $19 / 22(86)$ \\
\hline
\end{tabular}

${ }^{a}$ Studies presented here are not head-to-head trials designed or statistically powered to compare the efficacy or safety of pegloticase alone or in combination with immunomodulation

${ }^{\mathrm{b}}$ All patients received a biweekly infusion of pegloticase $8 \mathrm{mg}$ in combination with the immunomodulatory therapy shown

${ }^{\mathrm{c}}$ One patient received oral methotrexate 14 days after the initial pegloticase infusion

${ }^{\mathrm{d}}$ Excludes 2 patients for whom treatment is ongoing

$I M T$ immunomodulatory therapy

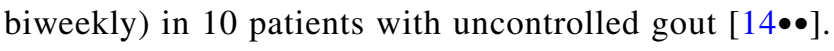
All 10 patients achieved a full therapeutic response and reached the primary end point, defined as the proportion of patients who maintained SU levels $<6.0 \mathrm{mg} / \mathrm{dL}$ for $80 \%$ of the time between months 3 and 6 . In addition, all patients completed full courses of treatment without increases in

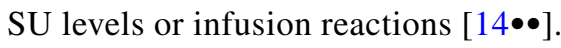

Albert et al. [8] conducted a case series in 10 patients with uncontrolled gout where administration of oral or subcutaneous MTX with pegloticase led to an $80 \%$ response rate. Nine of 10 patients received weekly injections of subcutaneous MTX (25 mg); 1 patient $12.5 \mathrm{mg}$ orally weekly. Two patients discontinued therapy before receiving the full 12-week infusion course: 1 due to loss of response and a mild infusion reaction and 1 to personal preference [8].

Bessen et al. [10] reported 7 patients with uncontrolled gout treated with MTX, AZA, or the immunosuppressive cyclosporine with pegloticase, initiated on the first day of infusions. Six of 7 received MTX; 1 patient was switched to azathioprine at the fifth pegloticase infusion due to fatigue related to MTX. Cyclosporine (50 mg twice daily [BID]) was chosen for 1 other patient due to elevated liver enzymes at baseline. All patients maintained SU levels $\leq 6 \mathrm{mg} / \mathrm{dL}$ (100\% response), and no infusion reactions were reported [10].

To assess responses in patients who received LEF coadministration with pegloticase, Masri et al. [12] retrospectively studied 10 with uncontrolled gout. Of 6 patients analyzed at the time of data cutoff, 4 met the primary end point (defined as those who received $\geq 12$ pegloticase infusions), and 2 were lost to follow-up, yielding a $67 \%$ overall response rate [12].

RECIPE (Reducing Immunogenicity to Pegloticase), an investigator-initiated proof-of-concept RCT, examined whether a short course of MMF could effectively and safely reduce immunogenicity to pegloticase [40••]. Patients with uncontrolled gout were randomized 3:1 to receive either $1 \mathrm{~g}$ MMF BID $(n=22)$ or placebo $(n=10)$, with a 2 -week runin prior to initiating pegloticase biweekly. After 12 weeks, patients in the MMF arm had MMF withdrawn and both arms were followed for another 12 weeks. Eighty-six percent (19 of 22) in the MMF arm achieved the primary endpoint of $\mathrm{SU} \leq 6 \mathrm{mg} / \mathrm{dL}$ at 12 weeks, compared with $40 \%$ (4 of 10 ) in placebo. At 24 weeks, serum uric acid response was sustained in $68 \%$ of the patients in the MMF arm, whereas the response rate for the placebo group further decreased to $30 \%$. The proportion of patients with sustained SU levels $<6 \mathrm{mg} / \mathrm{dL}$ at both 12 and 24 weeks was significantly higher with MMF in both groups $(p=0.02$ and $p=0.03$, respectively). Furthermore, there were no infusion reactions in the MMF group compared with $30 \%$ in patients receiving

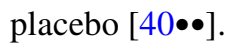

MIRROR-OL (Methotrexate to Increase Response Rates in Patients with Uncontrolled Gout Receiving Pegloticase), an open-label pilot study in adult patients with uncontrolled gout $(N=14)$, further evaluated the use of MTX with pegloticase [15]. The primary end point was the proportion of responders, defined as those who maintained SU levels $<6 \mathrm{mg} / \mathrm{dL} \geq 80 \%$ of the time during month 6 of pegloticase treatment [15]. Preliminary results showed a response rate of $78.6 \%(n=11 / 14)$, based on a 
Fig. 1 Proportion of patients receiving pegloticase and concomitant immunomodulatory therapy by year (2015-2019) [45•]. ${ }^{\text {a Patients who started }}$ either methotrexate or azathioprine within 60 days of their first pegloticase infusion

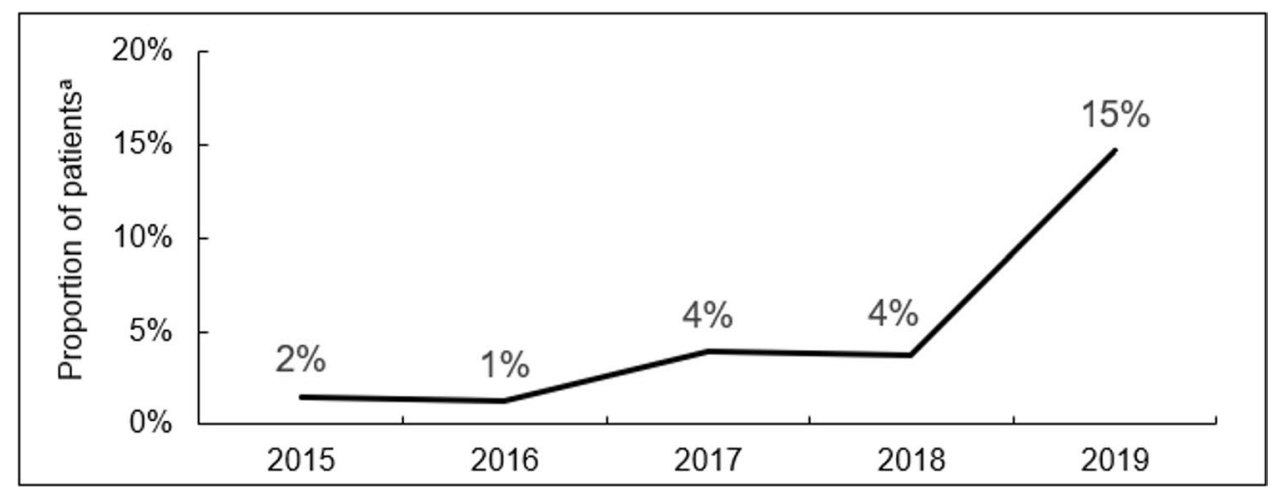

modified intention-to-treat population, defined as those who received $\geq 1$ pegloticase infusion. One investigator-reported infusion reaction, identified as a mild cough, occurred in a patient during their fifth pegloticase infusion; however, this patient still completed 24 weeks of treatment as a responder. Responders at 6 months who continued treatment $(n=8)$ remained responders at month 12 [15].

TRIPLE (Tolerization Reduces Intolerance to Pegloticase and Prolongs the Urate Lowering Effect) included patients with uncontrolled gout cotreated with AZA and pegloticase $(N=12)$; results were reported in an interim analysis. Patients received $1.25 \mathrm{mg} / \mathrm{kg}$ AZA daily for 1 week, increased to $2.5 \mathrm{mg} / \mathrm{kg}$ before the first pegloticase infusion, followed by $2.5 \mathrm{mg} / \mathrm{kg}$ daily for the trial duration [13]. To date, 6 patients have completed the treatment course, and 2 remain on treatment, all with persistent urate-lowering effects resulting in levels below the predefined SU threshold $(<6 \mathrm{mg} / \mathrm{dL})$. Of the remaining 4 patients not included in the analysis, 2 lost uratelowering efficacy, 1 experienced an infusion reaction during the first dose, and 1 had subjective intolerance to AZA [13].

PROTECT (Prospective Study of Pegloticase in Transplant Patients), a phase 4, multicenter, open-label study, evaluated the efficacy of pegloticase in adults with uncontrolled gout who underwent kidney transplant and were receiving post transplantation immunosuppressant therapy [41•]. Results from the completed trial demonstrated an $88.9 \%$ response rate (16/18 patients), with 2 patients removed due to COVID infection/COVID concerns [42].

Finally, MIRROR-RCT (Methotrexate to Increase Response Rates in Patients with Uncontrolled Gout Receiving Pegloticase), a multicenter, randomized, double-blind, placebo-controlled study in adult patients with uncontrolled gout showed a $71 \%$ response rate in the methotrexate-treated group vs. $38.5 \%$ in the placebo group with the primary endpoint defined as the proportion of responders who maintained SU levels $<6 \mathrm{mg} / \mathrm{dL}$ at least $80 \%$ of the time during month 6 [43].

A summation of a number of the previously described cases was included in a systematic review of 10 publications (describing 82 cases) using immunomodulation in the setting of pegloticase administration that demonstrated a marked improvement in responder rates (to $82.9 \%$ overall), compared with pegloticase monotherapy. Of the 80 cases on a single immunomodulatory agent, response rates of

Table 2 Immunomodulator considerations for use

\begin{tabular}{|c|c|c|c|}
\hline \multirow[t]{2}{*}{ Immunomodulator } & \multicolumn{3}{|l|}{ Considerations } \\
\hline & Hepatic & Renal & Other \\
\hline Methotrexate [48] & $\begin{array}{l}\text { - Avoid in preexisting liver disease } \\
\text { - Toxic interaction with alcohol } \\
\text { - Monitor CBCs and LFTs }\end{array}$ & $\begin{array}{l}\text { - Dose adjustment for renal impair- } \\
\text { ment }\end{array}$ & - Contraindicated with pregnancy \\
\hline Leflunomide [49] & $\begin{array}{l}\text { - Contraindicated with hepatic } \\
\text { impairment } \\
\text { - Liver function monitoring }\end{array}$ & $\begin{array}{l}\text { - Patients with a history of renal } \\
\text { failure or transplant may already } \\
\text { be taking this medication }\end{array}$ & - Contraindicated with pregnancy \\
\hline Mycophenolate mofetil [50] & $\begin{array}{l}\text { - Dose adjustment for adult trans- } \\
\text { plant patients } \\
\text { - Monitor CBCs and LFTs }\end{array}$ & $\begin{array}{l}\text { Patients with a history of renal } \\
\text { transplant or lupus nephritis may } \\
\text { already be taking this medication }\end{array}$ & $\begin{array}{l}\text { - Potential for severe and limiting GI, } \\
\text { glucose, and cholesterol side effects } \\
\text { - Contraindicated with pregnancy }\end{array}$ \\
\hline Azathioprine [47] & $\begin{array}{l}\text { - Potentially hepatotoxic in trans- } \\
\text { plant patients } \\
\text { - Monitor CBCs and LFTs }\end{array}$ & $\begin{array}{l}\text { - Dose adjustment for renal impair- } \\
\text { ment }\end{array}$ & $\begin{array}{l}\text { - Drug-drug interaction with allopu- } \\
\text { rinol } \\
\text { - Prescreen for TPMT }\end{array}$ \\
\hline
\end{tabular}

$C B C$ complete blood cell count; GI, gastrointestinal, $L F T$ liver function test, TPMT thiopurine S-methyltransferase 
90.3\% for oral MTX, $86.4 \%$ for MMF, $77.8 \%$ for subcutaneous MTX, 66.7\% LEF, and 63.6\% AZA [44]. Although it should be noted the case numbers are small and not designed to make comparisons between agents. As pegloticase is the only medication indicated for treatment of uncontrolled gout and often implemented as a last resort, maximizing the efficacy of such therapy is of paramount importance. As reviewed, practicing physicians, including authors of this publication, have opted to initiate immunomodulatory therapy before or in conjunction with pegloticase therapy to attenuate ADA formation and increase the potential for more durable responses. A retrospective claims analysis detailing the proportion of patients receiving concomitant immunomodulatory therapy with pegloticase by year (2015-2019) is shown in Fig. 1 [45•] with a dramatic increase observed following an ACR 2018 presentation detailing use of antiproliferative immunomodulators with pegloticase [45•].

\section{Considerations}

Patients with uncontrolled gout often have numerous comorbidities, including renal dysfunction, cardiovascular disease and a history of alcohol use or liver disease, and rheumatologists considering the use of an immunomodulator with pegloticase have several agents from which to choose [18, 46-49]. The availability of multiple antiproliferative therapies provides the flexibility to tailor therapy to the specific patient, crucial in those with multiple comorbidities requiring dose adjustments, hematologic testing, and/or other risk management measures (Table 2) [47-50]. Although the addition of these immunomodulators may increase risk for infection, their use is only temporary during active treatment and studies thus far have not shown an increased infection rate [47-50]. Data from the pending MIRROR-RCT

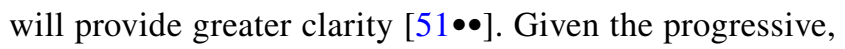
often erosive nature of tophaceous gout and limited treatment options, it is critical to consider implementation of one of these immunomodulatory agents in conjunction with pegloticase to maximize the likelihood of a full and durable response to therapy [18].

\section{Conclusions}

Based on the review of an accumulating body of evidence, concomitant immunomodulatory therapy administered with pegloticase can significantly improve durability of response, efficacy, and safety among patients with uncontrolled gout, for whom this may be the only treatment option. Current data from observational case studies, MIRROR-OL and RECIPE, demonstrate the consistency of these responses. Given the widespread use of antiproliferative agents to abrogate immunogenicity with other biologic agents for the treatment of rheumatic diseases, clinical rheumatologists should feel comfortable with similar treatment regimens with use of pegloticase for treatment of uncontrolled gout.

Acknowledgements We acknowledge Brian LaMoreaux, MD, MS, at Horizon Therapeutics, for his expertise and assistance throughout all aspects of the development of this publication.

Editorial support, under the guidance of the authors, was provided by Melissa Baire, MPH, MS, and Timothy Bayley, DO, PhD, CMPP, at The Lockwood Group (Stamford, CT, USA) in accordance with Good Publication Practice (GPP3) guidelines.

Author Contribution All authors had full access to all the data in this publication and take complete responsibility for the integrity of the data and accuracy of the data analysis.

Funding This manuscript was supported by funding from Horizon Therapeutics plc (Deerfield, IL). John K. Botson reports personal fees from Horizon Therapeutics, outside the submitted work.

\section{Compliance with Ethics Standards}

Conflict of Interest Dr. Botson has received research support from Horizon Therapeutics and Allena Pharmaceuticals as a principal investigator. He has received consulting/speaker fees from Horizon Therapeutics. He has participated on a Data Safety Monitoring Board/ Advisory Board for Horizon Therapeutics.

Dr. Baraf has received consulting/speaker fees from Horizon Therapeutics, and research grants or support from Horizon Therapeutics as an investigator.

Dr. Keenan has been or is a consultant with Horizon Therapeutics, Arthrosi Therapeutics, Selecta Biosciences, Atom Biosciences, and Sobi Pharma. He has received honoraria/speaker fees from Horizon Therapeutics.

Dr. Albert serves on a speaker bureau for Horizon Therapeutics. He has received consulting/speaker fees from Horizon Therapeutics. He has received travel support from Horizon Therapeutics. He has participated on an advisory board for Horizon Therapeutics.

Dr. Masri has been or is a consultant with Guidepoint Global. He has received speaker fees/honoraria from Horizon Therapeutics, Eli Lilly, and Pfizer. He has received payment for expert testimony from Cantor $\&$ Associates. He has participated on an advisory board for Horizon Therapeutics. He owns stock/stock options in Horizon Therapeutics, Gilead, Corbus Pharmaceutical, and Aurinia Pharma.

Dr. Peterson has received consulting/speaker fees from Horizon Therapeutics. He has participated on an advisory board for Horizon Therapeutics. He reports other financial/non-financial interests from Horizon Therapeutics.

Dr. Yung has received consulting/speaker fees from Horizon Therapeutics. She has participated on an advisory board for Horizon Therapeutics.

Dr. Freyne declares no conflict of interest.

Dr. Amin has received speaker fees/honoraria from Horizon Therapeutics and Eli Lilly.

Dr. Abdellatif has received consulting/speaker fees from Horizon Therapeutics. He has received payment for expert testimony from Horizon Therapeutics. He has participated on an advisory board for Horizon Therapeutics. He has received travel support from Horizon Therapeutics.

Dr. Nehad Soloman serves on a speaker bureau for Horizon Therapeutics. 
Dr. N. Lawrence Edwards has received consulting fees from Horizon Therapeutics, Shanton Pharma, and Atom Biosciences and Pharmaceuticals. He has received payment for expert testimony from Takeda Pharmaceuticals.

Dr. Strand reports consulting fees from AbbVie, Amgen, Arena, Aria, AstraZeneca, BMS, Boehringer Ingelheim, Celltrion, CORRONA, Crescendo/Myriad, EMD Serono, Equillium, Flexion, Galapagos, Genentech/Roche, Gilead, GSK, Horizon, Ichnos, Inmedix, Janssen, Kiniksa, Eli Lilly, Merck, MiMedx, Novartis, Pfizer, Regeneron, Rheos, R-Pharma, Samsung, Sandoz, Sanofi, Scipher, Servier, Setpoint, Sun Pharma, and UCB, outside the submitted work.

Human and Animal Rights and Informed Consent This article does not contain any studies with human or animal subjects performed by any of the authors.

Open Access This article is licensed under a Creative Commons Attribution 4.0 International License, which permits use, sharing, adaptation, distribution and reproduction in any medium or format, as long as you give appropriate credit to the original author(s) and the source, provide a link to the Creative Commons licence, and indicate if changes were made. The images or other third party material in this article are included in the article's Creative Commons licence, unless indicated otherwise in a credit line to the material. If material is not included in the article's Creative Commons licence and your intended use is not permitted by statutory regulation or exceeds the permitted use, you will need to obtain permission directly from the copyright holder. To view a copy of this licence, visit http://creativecommons.org/licenses/by/4.0/.

\section{References}

Papers of particular interest, published recently, have been highlighted as:

- Of importance

• Of major importance

1. Strand V, et al. Immunogenicity of biologics in chronic inflammatory diseases: a systematic review. BioDrugs. 2017;31:299316. https://doi.org/10.1007/s40259-017-0231-8.

2. Strand, V. et al. Immunogenicity of biosimilars for rheumatic diseases, plaque psoriasis, and inflammatory bowel disease: a review from clinical trials and regulatory documents. BioDrugs. 2020;34:27-37. https://doi.org/10.1007/s40259-019-00394-x. High-quality review and discussion on the immunogenicity of biosimilars.

3. Ben-Horin S, et al. Addition of an immunomodulator to infliximab therapy eliminates antidrug antibodies in serum and restores clinical response of patients with inflammatory bowel disease. Clin Gastroenterol Hepatol. 2013;11:444-7. https://doi.org/10. 1016/j.cgh.2012.10.020.

4. Ducourau, E. et al. Methotrexate effect on immunogenicity and long-term maintenance of adalimumab in axial spondyloarthritis: a multicentric randomised trial. RMD Open. 2020;6:doi:https://doi.org/10.1136/rmdopen-2019-001047.

5. Krieckaert CL, Nurmohamed MT, Wolbink GJ. Methotrexate reduces immunogenicity in adalimumab treated rheumatoid arthritis patients in a dose dependent manner. Ann Rheum Dis. 2012;71:1914-5. https://doi.org/10.1136/annrh eumdis-2012-201544.

6. Ong DE, Kamm MA, Hartono JL, Lust M. Addition of thiopurines can recapture response in patients with crohn's disease who have lost response to anti-tumor necrosis factor monotherapy. $\mathbf{J}$ Gastroenterol Hepatol. 2013;28:1595-9. https://doi.org/10.1111/ jgh.12263.

7. Maneiro JR, Salgado E, Gomez-Reino JJ. Immunogenicity of monoclonal antibodies against tumor necrosis factor used in chronic immune-mediated inflammatory conditions: systematic review and meta-analysis. JAMA Intern Med. 2013;173:141628. https://doi.org/10.1001/jamainternmed.2013.7430.

8. Albert JA, Hosey T, LaMoreaux B. Increased efficacy and tolerability of pegloticase in patients with uncontrolled gout co-treated with methotrexate: a retrospective study. Rheumatol Ther. 2020;7:639-48. https://doi.org/10.1007/s40744-020-00222-7.

9. Berhanu AA, Krasnokutsky S, Keenan RT, Pillinger MH. Pegloticase failure and a possible solution: immunosuppression to prevent intolerance and inefficacy in patients with gout. Semin Arthritis Rheum. 2017;46:754-8. https://doi.org/10. 1016/j.semarthrit.2016.09.007.

10. Bessen M.Y., Bessen S. Y. \& Yung, C. M. Concomitant immunosuppressant use with pegloticase in patients with tophaceous gout- a case series. Int J Clin Rheumatol. 2019;14(6).

11. Freyne B. A case report of immunosuppressant medicationassociated polyarticular tophaceous gout successfully treated using the polyethylene glycol-conjugated uricase enzyme pegloticase. Transplantation Proc. 2018;50:4099-101. https:// doi.org/10.1016/j.transproceed.2018.03.010.

12. Masri K, Winterling K, Lamoreaux B. Leflunomide co-therapy with pegloticase in uncontrolled gout. Ann Rheum Dis. 2020;79:454-454. https://doi.org/10.1136/annrheumdis-2020eular.3891.

13. Rainey H, Baraf HS, Yeo AE, Lipsky PE. Companion immunosuppression with azathioprine increases the frequency of persistent responsiveness to pegloticase in patients with chronic refractory gout. Ann Rheum Dis. 2020;79:438.

14. • Botson JK, Peterson J. Pretreatment and coadministration with methotrexate improved durability of pegloticase response: an observational, proof-of-concept case series. J ClinRheumatol. 2020. https://doi.org/10.1097/RHU.0000000000001639. First methodical proof-of-concept exploration of pegloticase with immunomodulation.

15. Botson J, et al. Pegloticase response improvement by co-treatment with methotrexate: results from the MIRROR open-label clinical trial in patients with uncontrolled gout. Ann Rheum Dis. 2020;79:442.

16. Chen-Xu M, Yokose C, Rai SK, Pillinger MH, Choi HK. Contemporary prevalence of gout and hyperuricemia in the united states and decadal trends: the national health and nutrition examination survey, 2007-2016. Arthritis Rheumatol. 2019;71:991-9. https://doi.org/10.1002/art.40807.

17. Edwards NL. Treatment-failure gout: a moving target. Arthritis Rheumatol. 2008;58:2587-90. https://doi.org/10.1002/art. 23803.

18. Terkeltaub, R. Gout: diagnosis and management of gouty arthritis and hyperuricemia. 3rd edn. (Professional Communications, Inc, 2013).

19. Ao J, Goldblatt F, Casson RJ. Review of the ophthalmic manifestations of gout and uric acid crystal deposition. Clin Exp Ophthalmol. 2017;45:73-80. https://doi.org/10.1111/ceo. 12749.

20. Klauser AS, et al. Dual-energy computed tomography detection of cardiovascular monosodium urate deposits in patients with gout. JAMA Cardiol. 2019;4:1019-28. https://doi.org/10. 1001/jamacardio.2019.3201.

21. Nickeleit V, Mihatsch MJ. Uric acid nephropathy and endstage renal disease-review of a non-disease. Nephrol Dial Transplant. 1997;12:1832-8. https://doi.org/10.1093/ndt/12.9. 1832 . 
22. Pascart, T. et al. Association of specific comorbidities with monosodium urate crystal deposition in urate-lowering therapy-naive gout patients: a cross-sectional dual-energy computed tomography study. J Clin Med. 2020;9:doi:https://doi.org/10.3390/jcm9051295.

23. Bongartz T, et al. Dual-energy CT for the diagnosis of gout: an accuracy and diagnostic yield study. Ann Rheum Dis. 2015;74:1072-7. https://doi.org/10.1136/annrh eumdis-2013-205095.

24. Doghramji PP, Wortmann RL. Hyperuricemia and gout: new concepts in diagnosis and management. Postgrad Med. 2012;124:98-109. https://doi.org/10.3810/pgm.2012.11.2616.

25. Thiele RG, Schlesinger N. Diagnosis of gout by ultrasound. Rheumatology (Oxford). 2007;46:1116-21. https://doi.org/10. 1093/rheumatology/kem058.

26. FitzGerald, J. D. et al. 2020 American College of Rheumatology guideline for the management of gout. Arthritis Care Res (Hoboken). 2020. doi:https://doi.org/10.1002/acr.24180

27. Dalbeth N, et al. Presence of monosodium urate crystal deposition by dual-energy CT in patients with gout treated with allopurinol. Ann Rheum Dis. 2018;77:364-70. https://doi.org/10.1136/ annrheumdis-2017-212046.

28. Mandell BF, Yeo AE, Lipsky PE. Tophus resolution in patients with chronic refractory gout who have persistent urate-lowering responses to pegloticase. Arthritis Res Ther. 2018;20:286. https://doi.org/10.1186/s13075-018-1782-x.

29. Perez-Ruiz F, Calabozo M, Erauskin GG, Ruibal A, HerreroBeites AM. Renal underexcretion of uric acid is present in patients with apparent high urinary uric acid output. Arthritis Rheum. 2002;47:610-3. https://doi.org/10.1002/art.10792.

30. Baraf HS, et al. Tophus burden reduction with pegloticase: results from phase 3 randomized trials and open-label extension in patients with chronic gout refractory to conventional therapy. Arthritis Res Ther. 2013;15:R137. https://doi.org/10. 1186/ar4318.

31. Baraf HS, Yood RA, Ottery FD, Sundy JS, Becker MA. Infusionrelated reactions with pegloticase, a recombinant uricase for the treatment of chronic gout refractory to conventional therapy. J Clin Rheumatol. 2014;20:427-32. https://doi.org/10.1097/RHU. 0000000000000200

32. Cunha RN, Aguiar R, Farinha F. Impact of pegloticase on patient outcomes in refractory gout: current perspectives. Open Access Rheumatol. 2018;10:141-9. https://doi.org/10.2147/OARRR. S176951.

33. Keenan RT, Baraf HSB, LaMoreaux B. Use of pre-infusion serum uric acid levels as a biomarker for infusion reaction risk in patients on pegloticase. Rheumatol Ther. 2019;6:299-304. https://doi.org/10.1007/s40744-019-0151-9.

34. Strand V, Khanna D, Singh JA, Forsythe A, Edwards NL. Improved health-related quality of life and physical function in patients with refractory chronic gout following treatment with pegloticase: evidence from phase iii randomized controlled trials. J Rheumatol. 2012;39:1450-7. https://doi.org/10.3899/ jrheum.111375.

35. Lipsky PE, et al. Pegloticase immunogenicity: the relationship between efficacy and antibody development in patients treated for refractory chronic gout. Arthritis Res Ther. 2014;16:R60. https://doi.org/10.1186/ar4497.

36. KRYSTEXXA (pegloticase injection) [prescribing information]. Horizon therapeutics.

37. Sundy JS, et al. Efficacy and tolerability of pegloticase for the treatment of chronic gout in patients refractory to conventional treatment: two randomized controlled trials. JAMA. 2011;306:711-20. https://doi.org/10.1001/jama.2011.1169.

38. Schlesinger N, Edwards NL, Yeo AE, Lipsky PE. Development of a multivariable improvement measure for gout. Arthritis Res Ther. 2020;22:164. https://doi.org/10.1186/s13075-020-02254-4.
39. Calabrese LH, Kavanaugh A, Yeo AE, Lipsky PE. Frequency, distribution and immunologic nature of infusion reactions in subjects receiving pegloticase for chronic refractory gout. Arthritis Res Ther. 2017;19:191. https://doi.org/10.1186/ s13075-017-1396-8.

$40 . \bullet$ Khanna, D. \& Khanna, P. P. Reducing immunogenicity of pegloticase (RECIPE) with concomitant use of mycophenolate mofetil in patients with refractory gout- results of a phase ii double blind randomized controlled trial. 2020. Early phase II randomized controlled trial demonstrating improved response rates in patients with uncontrolled gout treated with pegloticase and immunomodulation.

41. Abdellatif, A. et al. A multicenter, open-label, efficacy and safety study of pegloticase in patients with uncontrolled gout who have undergone kidney transplantation: early data report. Arthritis Rheumatol. 2020;72. Early open-label study demonstrating efficacy and safety of pegloticase and immunomodulation in renal transplant patients with uncontrolled gout.

42. Results from PROTECT Trial Evaluating KRYSTEXXA® (pegloticase injection) in kidney transplant recipients with uncontrolled gout presented as Part of American Society of Nephrology (ASN) Kidney Week. Horizon Therapeutics, 4 Nov. 2021, https://ir.horizontherapeutics.com/news-releases/newsrelease-details/results-protect-trial-evaluating-krystexxar-peglo ticase. Press release.

43. Horizon Therapeutics plc Submits Supplemental Biologics License for the Concomitant Use of KRYSTEXXA® (pegloticase injection) Plus Methotrexate for People Living with Uncontrolled Gout. Horizon Therapeutics, 10 Jan. 2022, https://ir.horiz ontherapeutics.com/news-releases/news-release-details/horizontherapeutics-plc-submits-supplemental-biologics-license.

44. Keenan RT, et al. The effect of immunomodulators on the efficacy and tolerability of pegloticase: a systematic review. Semin Arthritis Rheum. 2021;51:347-52. https://doi.org/10.1016/j. semarthrit.2021.01.005.

45. LaMoreaux, B., Francis-Sedlak, M., Svensson, K. \& Holt, R. Immunomodulation co-therapy with pegloticase: database trends 2014-2019. Ann Rheum Dis. 2020;79:108. Retrospective database analysis showcasing the increasing trend in immunomodulation use with pegloticase over a 5-year period.

46. Yood RA, Ottery FD, Irish W, Wolfson M. Effect of pegloticase on renal function in patients with chronic kidney disease: a post hoc subgroup analysis of 2 randomized, placebo-controlled, phase 3 clinical trials. BMC Res Notes. 2014;7:54. https://doi. org/10.1186/1756-0500-7-54.

47. Imuran (azathioprine) [prescribing information]. Sebela.

48. Methotrexate [prescribing information]. Hospira.

49. Arava (leflunomide) [prescribing information]. Sanofi-Aventis.

50. Cellcept (mycophenolate mofetil) [prescribing information]. Genentech.

51.•• Botson, J. K. et al. Pegloticase in combination with methotrexate in patients with uncontrolled gout: a multicenter, open-label study (MIRROR). J Rheumatol. 2021;48:767-774. https://doi. org/10.3899/jrheum.200460. Open-label trial demonstrating 6-month sustained therapeutic response after combination therapy with pegloticase and methotrexate compared to pegloticase monotherapy.

Publisher's Note Springer Nature remains neutral with regard to jurisdictional claims in published maps and institutional affiliations. 


\section{Authors and Affiliations}

\section{John K. Botson ${ }^{1}\left[\right.$. Herbert S. B. Baraf ${ }^{2} \cdot$ Robert T. Keenan $^{3} \cdot$ John Albert $^{4} \cdot$ Karim R. Masri $^{5} \cdot$ Jeff Peterson $^{6}$. Christianne Yung $^{7} \cdot$ Brigid Freyne $^{8} \cdot$ Mona Amin $^{9} \cdot$ Abdul Abdellatif $^{10} \cdot$ Nehad Soloman $^{11} \cdot$ N. Lawrence Edwards ${ }^{12}$. Vibeke Strand ${ }^{13}$}

Herbert S. B. Baraf

hsbbaraf@mac.com

Robert T. Keenan

robert.keenan@duke.edu

John Albert

John.albert@rdcwi.com

Karim R. Masri

karimmasri@rheumondemand.com

Jeff Peterson

jeff.peterson@wwmedgroup.com

Christianne Yung

arthritisoffice@aol.com

Brigid Freyne

brigid.freyne@brigidfreyne.com

Mona Amin

mksmona@gmail.com

Abdul Abdellatif

Abdul71md@yahoo.com

Nehad Soloman

rheumboy@yahoo.com

N. Lawrence Edwards

edwarnl@medicine.ufl.edu

Vibeke Strand

vstrand@stanford.edu

1 Orthopedic Physicians Alaska 3801 Lake Otis Pkwy, Anchorage, AK 99508, USA
2 The Center for Rheumatology and Bone Research, 2730 University Blvd. West, Suite 310, Wheaton, MD 20902, USA

3 Duke University School of Medicine Duke Medicine Circle, 124 Davison Building, Durham, NC 27710, USA

4 Rheumatic Disease Center, 7080 N. Port Washington Road, Glendale, WI 53217, USA

5 Rheumatology OnDemand, LLC 405 Welwyn Rd, Henrico, VA 23229, USA

6 The Seattle Arthritis Clinic, Kirkland, WA 98033, USA

7 Private Practice, 2482 W Horizon Ridge Parkway, Suite 130, Henderson, NV 89052, USA

8 Rheumatology Internal Medicine 39755, Murrieta Hot Springs Rd, Ste. F110, Murrieta, CA 92563, USA

9 Arizona Arthritis and Rheumatology Associates, 11943 East Beryl Ave, Scottsdale, AZ 85259, USA

10 Baylor College of Medicine, 600 N Kobayashi Rd., Ste 312, Webster, TX 77598, USA

11 Arizona Arthritis and Rheumatology Associates, 9097 W Roberta Ln, Phoenix, AZ 85383, USA

12 University of Florida, 1600 SW Archer Road, Room 4102, Gainesville, FL 32610, USA

13 Division of Immunology/Rheumatology, Stanford University, 306 Ramona Road, Portola Valley, CA 94028, USA 